\title{
Robust Joint Expansion Planning of Electrical Distribution Systems and EV Charging Stations
}

\author{
Nataly Bañol Arias, Alejandra Tabares, John F. Franco ${ }^{(0)}$, Member, IEEE, Marina Lavorato, Member, IEEE, \\ and Rubén Romero ${ }^{(0)}$, Senior Member, IEEE
}

\begin{abstract}
Electrical distribution systems (EDSs) should be prepared to cope with demand growth in order to provide a quality service. The future increase in electric vehicles (EVs) represents a challenge for the planning of the EDS due to the corresponding increase in the load. Therefore, methods to support the planning of the EDS, considering the uncertainties of conventional loads and EV demand, should be developed. This paper proposes a mixedinteger linear programming (MILP) model to solve the robust multistage joint expansion planning of EDSs and the allocation of EV charging stations (EVCSs). Chance constraints are used in the proposed robust formulation to deal with load uncertainties, guaranteeing the fulfillment of the substation capacity within a specified confidence level. The expansion planning method considers the construction/reinforcement of substations, EVCSs, and circuits, as well as the allocation of distributed generation units and capacitor banks along the different stages in which the planning horizon is divided. The proposed MILP model guarantees optimality by applying classical optimization techniques. The effectiveness and robustness of the proposed method is verified via two distribution systems with 18 and 54 nodes. Additionally, Monte Carlo simulations are carried out, aiming to verify the compliance of the proposed chance constraint.
\end{abstract}

Index Terms-Chance constraint, electrical distribution systems, electric vehicle charging stations, mixed-integer linear programming, multistage expansion planning.

\section{INTRODUCTION}

$\mathbf{T}$ HE use of Electric Vehicles (EVs) is expected to increase in the next few years as an option for resolving environment problems, such as climate change [1]. The adoption of EVs contributes to the reduction of air pollutant emissions and could take advantage of renewable energy sources when the EV batteries need to be charged. However, the electrical distribution system (EDS) should be prepared to contend with an increase in demand related to EV charging.

Manuscript received February 3, 2017; revised September 2, 2017; accepted October 13, 2017. Date of publication October 18, 2017; date of current version March 20, 2018. This work was supported by the Brazilian institutions CAPES, FAPESP, and CNPq Process 152002/2016-2. Paper no. TSTE-001072017. (Corresponding author: John F. Franco.)

N. Bañol Arias, A. Tabares, and R. Romero are with the Department of Electrical Engineering, São Paulo State University (UNESP), Ilha Solteira 15385-000, Brazil (e-mail: natycanta@gmail.com; tabares.1989@gmail.com; ruben@dee.feis.unesp.br).

J. F. Franco is with São Paulo State University (UNESP), Rosana 19274-000, Brazil (e-mail: j.f.franco@ieee.org).

M. Lavorato is with the CEATEC-Pontifical Catholic University of Campinas, Campinas 13086-900, Brazil (e-mail: marina.oliveira@pucampinas.edu.br).

Color versions of one or more of the figures in this paper are available online at http://ieeexplore.iee.org.

Digital Object Identifier 10.1109/TSTE.2017.2764080
Household charging is the first choice for EV owners, although the corresponding slow charging mode is timeconsuming [2]. On the other hand, electric vehicle charging stations (EVCSs) are a suitable option for EV charging, as they could avoid overloading residential distribution networks, while allowing both slow (preserving battery lifespan) and fast charging modes. EVCSs could also offer lower energy prices and reduce charging time (similar to the refueling of conventional vehicles) when compared to residential charging. These benefits will potentially encourage the use of EVs.

A high penetration of EVs in residential and commercial areas could result in operational problems, such as overloads, voltage issues, and excessive energy losses [3]. Therefore, the EDS expansion planning should satisfy the energy requirements of upcoming EV penetration for both household charging and EVCSs.

The solution of the EDS expansion planning problem identifies the investments needed to supply the future loads while satisfying operational constraints. This optimization problem is highly complex and NP-hard, due to the binary variables that represent the construction and/or allocation of new equipment and the high number of continuous variables used to represent the steady-state operation of the network. This problem has been widely studied, using different mathematical models and solution techniques. Nonetheless, the expansion planning of the EDS, considering the high penetration of EVs, needs to be studied further. A complete literature review of the EDS expansion planning problem can be found in [4].

Methods based on evolution algorithms and Mixed-Integer Linear Programming (MILP) have been developed to solve the EDS expansion planning problem considering the integration of EVs [5]-[8]. The allocation of EV battery charging/swap stations is carried out in [5] to minimize the costs related to reinforcement and adaption (construction costs necessary to cover the insufficiency of the plan). A method to solve the multistage EDS expansion planning problem, which takes into account the allocation and sizing of EVCSs, is proposed in [6]. Similarly, in [7] and [8], the joint expansion planning of EDS and EVCSs is addressed. In [7], the authors establish a bi-objective deterministic collective planning model for EDS that considers EVCSs, although the stochastic behavior of EV users, along with different EV charging modes, is disregarded. Moreover, the increase in the annual demand, impacts of the geographic locations, and time periods of fast- and slow-charging modes, in the expansion planning are not considered. In order to overcome those 
limitations, the authors in [8] propose a stochastic multistage collaborative planning model for EDS that considers EVCSs, slow- and fast-charging modes, and battery exchange. Nevertheless, the metaheuristic technique used to solve the problem does not provide information related to the quality of the solution (e.g., the distance from the obtained solution to the optimal solution), and there is not a way to define the level of confidence to address uncertainties related to demand profiles. On the other hand, the allocation of Distributed Generation (DG) units and Capacitor Banks (CB), is also disregarded in [5]-[8]. The installation of these equipment's should also be included in the expansion planning, as they could defer reinforcement, resulting in the reduction of investments [9].

The allocation and sizing problem of EVCSs is studied in [10]-[17]; nevertheless, these approaches solve the problem without considering the joint expansion planning of the whole grid. Reference [10] models the EVCS behavior, taking into account market interactions (reserve and energy markets), DG units operation and enforce network constraints in a two-stage approach. However, the proposed methodology in [10] is mostly focused on the EVCS operation planning. Similarly, in [11], a two-stage methodology to solve the optimal planning of EVCSs was proposed. Environmental factors, service radius, and different charger types are included in the proposed method; however, the authors disregarded the operation of both CBs and DG units, and the inclusion of different types of EVs. Metaheuristic techniques, such as genetic algorithms [12]-[14] and particle swarm optimization [16], [17], have also been used to solve the optimal allocation and sizing of EVCSs. From those works, only [17] considers the operation of DG units within the method. Moreover, in contrast to classical optimization techniques (such as those used in this paper), the main disadvantage of the metaheuristic techniques is that they do not guarantee optimal solutions.

Different from the previous works, authors in [18] present a new methodology for the optimal allocation of EVCSs based on a sustainability perspective. A multicriteria decision-making (MCDM) method, along with a fuzzy TOPSIS method, are used to take into account aspects related to economic growth, social development, and environmental protection. The EVCS allocation decision is carried out based on the criteria of five groups of expert panels, without considering mathematical models to represent the problem. This methodology allows for the consideration of quantitative and qualitative criteria, which are important in the allocation and sizing of EVCSs. However, from the EDS point of view, technical aspects of the grid operation should be verified.

Uncertainties associated with the growth of conventional loads and EV demand should be considered in the EDS expansion planning problem in order to reduce risks and avoid underinvestment, which could lead to operational problems. From the aforementioned references, [5], [10], [13], and [17] consider both the uncertain behavior of EVs as well as the operational constraints of the EDS, whereas [14] only considers the uncertain behavior of EVs. In [5], the uncertainty associated with $\mathrm{EV}$ demand is addressed using a geometric Brownian motion approach, while in [10], it is dealt with a two-stage stochastic programming model, along with an approach for generation of scenarios. Moreover, in [13] and [14], the authors represent the uncertainties of the EVs through probabilistic parameters and probability distribution functions for the arrival and SOC data, while in [17], the hourly aggregated load demand of EVs is estimated using a non-Gaussian multivariate stochastic model provided by copula functions. Nevertheless, none of those studies considers the uncertainty associated with the conventional loads.

Most of the aforementioned methods focus on modeling the allocation and sizing of the EVCSs based on their planning operation. In contrast to them, the main purpose of the proposed method is to consider the allocation and sizing of the EVCSs within the expansion planning of the EDS, in order to illustrate the impact of the EV integration in distribution systems, according to the EV connection point.

This paper presents a MILP model used to solve the robust multistage joint expansion planning of EDSs and the allocation of EVCSs. The proposed robust formulation uses chance constraints to deal with the uncertainties related to conventional loads and EV demand, guaranteeing the fulfillment of the substation capacity within a specified confidence level. The expansion planning method considers the construction/reinforcement of substations, EVCSs, and circuits, as well as the allocation of DG units and CBs along the different stages in which the planning horizon is divided. Similarly to [9], piecewise linearization was used to represent the square of active and reactive powers in the equations that model the steady-state operation of the EDS. Therefore, the proposed method is a MILP formulation that can be solved guaranteeing optimality using commercial solvers such as CPLEX. The main contributions of this paper relies on the application of

1) A chance constraint stochastic programming framework [19], which is suitable for addressing the uncertainties related to conventional loads and EV demand in the EDS expansion planning. This approach ensures the fulfillment of the substation capacity (the most important equipment of the network) within a confidence level, as was done in [20];

2) Linearizations and simplifications, formulated in order to consider the stochastic behavior of conventional loads and EV demand in the EDS expansion planning problem. In contrast to other works, which use methods based on chance constraint stochastic programming, the proposed formulation makes it possible to incorporate different network equipment, such as DG units and $\mathrm{CBs}$;

3) A mixed-integer linear formulation for the EDS expansion planning, which guarantees finite convergence to optimality while providing a measure of the distance to the optimum solution [21], and for which efficient software is available [22];

4) A novel analysis of the EV integration in distribution systems, which examines the impact of EV demand on the multistage expansion planning of EDS according to the connection point, along with a comparative analysis, which shows the benefits of including the uncertainty of 
the conventional loads and EV demand in the distribution planning when compared with deterministic models.

The effectiveness and robustness of the proposed method is verified via a 18 -node and 54-node test system. Additionally, Monte Carlo simulations are carried out, aiming to verify the compliance of the proposed chance constraint.

\section{Multistage Expansion PlanNing OF EDSs, CONSIDERING EVCSS}

As discussed in the previous section, the main goal of the EDS expansion planning is to adequately meet the load growth with a minimum total cost, subject to a set of technical and operational constraints. The complexity of the distribution planning has been increased over the past few years, due to the emergence of new network elements and the stochastic behavior of new loads (e.g., plug-in electric vehicles). In this context, the proposed method defines the investments needed to satisfy the load growth while keeping a suitable operation. The construction/reinforcement of substations and circuits is analyzed along different stages of the planning horizon, in an attempt to minimize the total investment and operational cost. In addition, DG units and CBs are considered, in order to improve the operation.

The expansion planning of the EDS should also take into account the allocation and sizing of EVCSs (from a set of suitable candidate nodes), which provide the energy required to charge EVs. Since previous works have focused on the operation of EVCSs without considering the expansion of the network [10][17], the proposed model is aimed toward a joint expansion planning of EDS and EVCSs. For this purpose, the operation of an EVCS and the corresponding EV charging process should be represented. However, the formulation of this charging process within the expansion planning is complex. Due to this fact, some details related to the operation of an EVCS are simplified within the formulation of the expansion planning. For instance, the EVCS operation issues (i.e., difference in the EV arrivals, waiting/idle times, and charging coordination) are simplified, assuming that the corresponding power is demanded in a typical day during a specific period of time (e.g., 12 hours), represented by $d w^{c s}$.

It is assumed that not all the EVs arrive at the EVCS at the same time; it is further assumed that the charging operation follows a "first come, first served" sequence. Thus, if an EV arrives while all of the chargers are in use, it has to wait until the next charger becomes available. The fact that the EVs have different states of charge (SOC) at arrival is represented by the factor $\phi_{s o c}$, which depends on each EV type, the daily distance driven, and other aspects related to the behavior of the EV owners. This factor is considered as the mean value of the SOC, which usually is represented by a Gaussian distribution function.

The proposed formulation identifies the optimal solution for the EVCSs, i.e., their location and the minimum number of chargers of each type required to meet the EV demand in each stage along the planning horizon. In order to represent the worst case for the EDS operation, it is assumed that the chargers are connected simultaneously and are demanding their rated power from the grid.
The following assumptions are made in order to formulate a mathematical model for the multistage EDS expansion planning problem considering EVCSs:

1) Given an EV penetration for each stage, the allocation and sizing of EVCSs is proposed by the distribution system operator, as considered in [5], [6], [10], [11]. This idea stems from the fact that the EDS must be prepared to satisfy the charging requirements resulting from the expected increase of the EV demand;

2) A fraction of the EVs are charged in the EVCSs, while the rest are charged at home, therefore, increasing the conventional load;

3) The EVs can be recharged using two charger types (fast or slow chargers).

Uncertainties related to the growth of conventional loads and EV demand are handled in the proposed model through chance constraints (see Section III-G), which guarantee the fulfillment of the substation capacity within a specified confidence level. It is assumed that the power related to the conventional loads and the number of EVs connected in the EDS are independent normal variables, as discussed in [23], i.e., there is not a correlation between the variables, which facilitates the calculation of the mean value and the standard deviation of the substation's apparent power.

\section{MATHEMATICAL Model}

The multistage EDS expansion planning problem can be mathematically formulated as a mixed-integer nonlinear programming (MINLP) model, which is highly complex to solve [24], [25]. Thus, linearization techniques can be used in order to transform the formulation into an MILP model. The proposed MILP formulation, which is based on [9], is used for solving the robust multistage joint expansion planning of EDSs and the allocation of EVCSs.

Due to the complexity of the EDS expansion planning and the relatively high substation investment cost, the proposed robust formulation uses chance constraints, which consider uncertainties related to conventional loads and EV demand, to enforce the capacities of the substations. The uncertainty of the conventional load and EV demand is modeled through a known normal distribution variable for the loads, as well as for the number of EVs that should be charged in each stage of the planning horizon. On the other hand, voltage and current limits are imposed in a deterministic way, i.e., mean values are assumed for the demands.

Equations that represent the influence of EVCSs on the EDS expansion planning, are presented in this section. Due to the lack of space, equations related to the operational limits of CBs and DG units, as well as the radiality conditions, are not shown. However, a complete definition of these constraints can be found in [9].

The following index will be used to represent the corresponding sets: $a, b, c$ for conductor types; $i$ for nodes; $h, t, r$ for substation alternatives; $e$ for charger types; $g$ for DG unit alternatives; $s$ for substation nodes; $i j, k j$ for circuits; $u, k$ for stages; $v$ for EV types; $p$ for EVCS nodes; and $m$ for DG unit nodes. 


\section{A. Objective Function}

The objective function minimizes the total expansion planning cost, comprising the investment, the operational, and the energy loss costs along the planning horizon. The investment costs in the circuits (IC), substations (IS), capacitors banks (ICB), and DG units (IDG) are calculated by (1)-(4), respectively. This set of equations is written in terms of the variables that represent the investment decisions. Thus, the binary investment variable $x_{i j, a, b, u}^{c i r}$ that represents the construction/reinforcement (using conductor type $b$ and assuming initial type $a$ ) is used to calculate the investment in the circuit, considering the conductor type cost $\left(c_{i j, a, b}^{c}\right)$ and its length $\left(l_{i j}\right)$. In a similar way, the cost related to the substations is calculated by using the binary variable for construction/reinforcement $x_{s, h, t, u}^{s u b}$ (using substation type $t$ and assuming initial type $h$ ) and the corresponding cost $c_{s, h, t}^{s}$. Moreover, the cost of CBs depends on the decision variables (binary for installation, $x_{i, u}^{c b}$, and integer for the number of standard capacitor units, $\left.n_{i, u}^{c b i}\right)$ and the corresponding costs ( $c^{c b}$ for installation and $c^{\text {mod }}$ per module). The investment in DG units is calculated according to the binary installation variable $x_{m, g, u}^{d g}$ and the installation cost $c_{g}^{d g}$.

The cost associated with the EVCS (ICS) is calculated by (5) in terms of the decision variables (binary for the allocation and integer for the number of chargers, $x_{p, u}^{c s}$ and $n_{p, e, u}^{c h i}$, respectively) and the installation costs (parameters $c^{c s}$ and $c_{e}^{c}$ for the EVCS and the chargers, respectively). Note that the maintenance cost and operational cost of the EVCS are not considered explicitly, although they can be included within the installation costs.

$$
\begin{aligned}
I C & =\sum_{i j} \sum_{a} \sum_{b} c_{i j, a, b}^{c} x_{i j, a, b, u}^{c i r} l_{i j} \\
I S & =\sum_{s} \sum_{h} \sum_{t} c_{s, h, t}^{s} x_{s, h, t, u}^{s u b} \\
I C B & =\sum_{i}\left(c^{c b} x_{i, u}^{c b}+c^{m o d} n_{i, u}^{c b i}\right) \\
I D G & =\sum_{m} \sum_{g} c_{g}^{d g} x_{m, g, u}^{d g} \\
I C S & =\sum_{p} \sum_{e}\left(c^{c s} x_{p, u}^{c s}+c_{e}^{c} n_{p, e, u}^{c h i}\right)
\end{aligned}
$$

The energy cost (EC) and the operational cost of substations (OS) are calculated by (6) and (7). The EC is written in terms of the cost of the energy imported by the substation $\left(c^{e}\right)$, the energy cost of the DG units $\left(c_{g}^{e d g}\right)$, and the corresponding active powers $P_{s, u}^{S}$ and $P_{m, u}^{G D}$, multiplied by the number of hours in one year $(\alpha)$ and the load factor $\left(\phi_{l}\right)$. The OS is calculated using the apparent power supplied by the substation that depends on the square approximation of the active and reactive power $\left(P_{i, u}^{S}\right.$ and $Q_{i, u}^{S}$, respectively), the operation cost $\left(c_{s}^{v}\right)$, and the loss factor $\phi_{s}$. Moreover, the function $f(\rho, \bar{\rho}, \Gamma)$ represents a piecewise linearization of the square value of a variable $\rho$, and it is written in terms of its maximum value $\bar{\rho}$ and the number of discretization intervals $\Gamma$, as described in the appendix.
The function $\zeta(\tau, K)=\left(1-(1+\tau)^{-K}\right) \tau^{-1}$ in (6) and (7) is used to calculate the present value of an annualized cost that has a duration of $K$ years in terms of the interest rate $\tau$. Thus, $K$ represents the number of years of each stage.

$$
\begin{aligned}
E C & =\alpha \phi_{l}\left(\sum_{s} c^{e} P_{s, u}^{S}+\sum_{m} \sum_{g} c_{g}^{e d g} P_{m, u}^{D G}\right) \zeta(\tau, K) \\
O S & =\sum_{s} \alpha \phi_{s} c_{s}^{v} \sum_{t}\left[f\left(P_{s, u}^{S}, \bar{S}_{t}, \Gamma\right)\right. \\
& \left.+f\left(Q_{s, u}^{S}, \bar{S}_{t}, \Gamma\right)\right] \zeta(\tau, K)
\end{aligned}
$$

Therefore, the objective function is defined as

$$
\min \sum_{u} \frac{I C+I S+I C B+I D G+I C S+E C+O S}{(1+\tau)^{-(u-1) K}}
$$

\section{B. Fundamental Constraints of the EDS}

The set of equations (9)-(12) corresponds to Kirchhoff's laws and represents the operation of radial EDSs [9]. Constraints (9) and (10) represent the active and reactive power balance, which guarantee that all loads are supplied, i.e., Kirchhoff's first law. Constraint (11) calculates the current magnitude throughout the circuit $i j$, while constraint (12) defines the voltage drop in the circuit $i j$ in terms of its connection status (represented by the binary variable $\left.y_{i j, a, u}^{c i r}\right)$, the active and reactive power flows $\left(P_{i j, a, u}\right.$ and $\left.Q_{i j, a, u}\right)$, and the square of the current $\left(I_{i j, a, u}^{s q r}\right)$. These variables are different from zero only if the corresponding conductor type $a$ is chosen, i.e., $y_{i j, a, u}^{c i r}$ is equal to one. Thus, (11) and (12) represent Kirchhoff's second law for each fundamental loop.

$$
\begin{aligned}
& \sum_{k j} \sum_{a} P_{k j, a, u}-\sum_{i j} \sum_{a}\left(P_{i j, a, u}+R_{a} l_{i j} I_{i j, a, u}^{s q r}\right)+P_{i, u}^{S} \\
& \quad+\sum_{g} P_{i, g, u}^{D G}=P_{i, u}^{D}+\sum_{e} n_{i, e, u}^{c h o} P_{e}^{c h} \forall i, u \\
& \sum_{k j} \sum_{a} Q_{k j, a, u}-\sum_{i j} \sum_{a}\left(Q_{i j, a, u}+X_{a} l_{i j} I_{i j, a, u}^{s q r}\right) \\
& \quad+n_{i, u}^{b c o} Q^{c b}+Q_{i, u}^{S}+\sum_{g} Q_{i, g, u}^{D G}=Q_{i, u}^{D} \quad \forall i, u \\
& \quad V_{j, u}^{\prime 2} I_{i j, a, u}^{s q r}=f\left(P_{i j, a, u}, \overline{V I}_{a}, \Gamma\right) \\
& \quad+f\left(Q_{i j, a, u}, \overline{V I}_{a}, \Gamma\right) \forall i j, a, u \\
& \quad V_{i, u}^{s q r}-V_{j, u}^{s q r}-\sum_{a}\left[2\left(R_{a} P_{i j, a, u}+X_{a} Q_{i j, a, u}\right) l_{i j}\right. \\
& \left.\quad+Z_{a}^{2} l_{i j}^{2} I_{i j, a, u}^{s q r}\right] \leq\left(\bar{V}^{2}-\underline{V}^{2}\right) \sum_{a}\left(1-y_{i j, a, u}^{c i r}\right) \quad \forall i j, u
\end{aligned}
$$

The EV demand is represented on the right-hand side of (9) as the product of the rated active power of each charger type $\left(P_{e}^{c h}\right)$ and the number of chargers operating in the corresponding EVCS $\left(n_{i, e, u}^{c h o}\right)$. The set of equations above uses the 
following variables and parameters: $P_{i, u}^{D}$ and $Q_{i, u}^{D}$ are the active and reactive power demands at node $i ; R_{a}, X_{a}$, and $Z_{a}$ are the resistance, reactance, and impedance per length of conductor type $a$, respectively; $n_{i, u}^{c b o}$ is the number of standard capacitor units operating at node $i$, while $Q^{c b}$ is the reactive power of each capacitor unit; $Q_{i, g, u}^{D G}$ is the reactive power supplied by the DG unit at node $i . \underline{V}$ and $\bar{V}$ are the lower and upper voltage limits, respectively, while $\bar{I}_{a}$ is the current limit of conductor type $a$. The parameter $V_{j, u}^{\prime}$ is the estimated voltage at node $i$, and it is used to obtain a linear expression on the left-hand side of (11), as proposed in [9].

Operational constraints, such as voltage and current limits in the system, are defined by (13) and (14), respectively.

$$
\begin{array}{r}
\underline{V}^{2} \leq V_{i, u}^{s q r} \leq \bar{V}^{2} \forall i, u \\
0 \leq I_{i j, a, u}^{s q r} \leq \bar{I}_{a}{ }^{2} y_{i j, a, u}^{c i r} \quad \forall i j, a, u
\end{array}
$$

\section{Logical Constraints Associated With Substations}

Constraints (15)-(19) allow for the coordination of the investment and operation of the substations along the planning horizon. The investment types correspond to the available apparent power capacities for the construction/reinforcement of the substations. In this way, the binary variable $x_{s, h, t, u}^{s u b}$ represents the option to construct/reinforce a substation using type $t$ from initial type $h$ (only transitions in which $t>h$ are allowed). Moreover, the substation types are sorted incrementally, according to the power capacity and the investment costs. Constraint (15) avoids the execution of more than one type of investment in the same stage (i.e., only one of the available power capacities can be chosen for construction/reinforcement), while (16) guarantees that a specific investment in a substation (from $h$ to $t$ ) can be carried out only one time along the planning horizon. In addition, (17) establishes that the reinforcement of a substation using initial type $h$ can be done only if that type was used to construct/reinforce the substation in previous stages. The binary parameter $\theta_{s, h}^{s u b}$ represents the initial state of the substation at the beginning of the planning horizon, i.e., it is 1 if the substation was constructed and 0 otherwise. Finally, (18) guarantees that the operation of a substation is enabled only if the corresponding investment was carried out, while (19) allows for the operation of the substation using only one type of investment in each stage, following the same logic as (15) for the operation state.

$$
\begin{aligned}
& \sum_{h} \sum_{t} x_{s, h, t, u}^{s u b} \leq 1 \forall s, u \\
& \sum_{u} x_{s, h, t, u}^{s u b} \leq 1 \forall s, h, t \\
& x_{s, h, t, u}^{s u b} \leq \theta_{s, h}^{s u b}+\sum_{k=1}^{u-1} \sum_{r} x_{s, r, h, k}^{s u b} \quad \forall s, h, t, u \\
& y_{s, t, u}^{s u b} \leq \theta_{s, t}^{s u b}+\sum_{k=1}^{u} \sum_{h} x_{s, h, t, k}^{s u b} \forall s, t, u \\
& \sum_{t} y_{s, t, u}^{s u b} \leq 1 \quad \forall s, u
\end{aligned}
$$

\section{Logical Constraints Associated With Circuits}

Constraints (20)-(24) enable the coordination of the investment and operation of the circuits along the planning horizon. This set of equations follows the same logical structure of the constraints related to the coordination of the investment and operation of the substations. The investment types correspond to the available current capacities for the construction/reinforcement of the circuits. In this way, the construction/reinforcement of a circuit using type $b$ from initial type $a$ is represented by the binary variable $x_{i j, a, b, u}^{c i r}$ (only transitions in which $b>a$ are allowed). The operation of a circuit using type $b$ is represented by the binary variable $y_{i j, b, u}^{c i r}$, and the binary parameter $\theta_{i j, a}$ represents the initial state of the circuit at the beginning of the planning horizon, i.e., it is 1 if the circuit was constructed and 0 otherwise.

$$
\begin{aligned}
& \sum_{a} \sum_{b} x_{i j, a, b, u}^{c i r} \leq 1 \quad \forall i j, u \\
& \sum_{u} x_{i j, a, b, u}^{c i r} \leq 1 \forall i j, a, b \\
& x_{i j, a, b, u}^{c i r} \leq \theta_{i j, a}^{c i r}+\sum_{k=1}^{u-1} \sum_{c} x_{i j, c, a, k}^{c i r} \forall i j, a, b, u \\
& y_{i j, b, u}^{c i r} \leq \theta_{i j, b}^{c i r}+\sum_{k=1}^{u} \sum_{a} x_{i j, a, b, u}^{c i r} \quad \forall i j, b, u \\
& \sum_{b} y_{i j, b, u}^{c i r} \leq 1 \quad \forall i j, u
\end{aligned}
$$

\section{E. Mathematical Modeling of EVCSs}

The set of equations (25)-(28) used to model the EVCSs was developed according to the assumptions established in Section II. Constraint (25) guarantees that an EVCS can be allocated only one time in a node along the planning horizon. In addition, (26) allows the installation of chargers (considering the maximum number $\bar{C}_{p}$ ) only if an EVCS was already allocated. Constraint (27) limits the number of chargers operating in each stage such that they do not exceed the number of chargers already installed.

$$
\begin{aligned}
& \sum_{u} x_{p, u}^{c s} \leq 1 \forall p \\
& \sum_{e} \sum_{k=1}^{u} n_{p, e, k}^{c h i} \leq \bar{C}_{p} \sum_{k=1}^{u} x_{p, k}^{c s} \forall p, u \\
& n_{p, e, u}^{c h o} \leq \sum_{k=1}^{u} n_{p, e, k}^{c h i} \forall p, e, u
\end{aligned}
$$

Equation (28) relates the number of EVs of type $v$ that need to be charged $\left(N_{v, u}^{E V}\right)$ with the number of EVs that are assigned to different charger types $\left(n_{e, v, u}^{e v}\right)$. The term on the right-hand side of (28) is used to consider the stochastic behavior associated with the number of EVs, and it depends on the robustness factor $\phi(\varepsilon)$ corresponding to the area under a normal distribution curve for a confidence level of $1-\varepsilon$ and the standard deviation of the number of EVs $\left(\sigma_{v, u}^{E V}\right)$. Moreover, (29) establishes that 
the energy that can be supplied by the chargers during their operating time $d w^{c s}$ should satisfy the energy required by the EVs. It is written in terms of the rated power of the charger type $e\left(P_{e}^{c h}\right)$, the energy required by an EV of type $v\left(E_{v}^{r e q}\right)$, the difference between the maximum EV SOC $\left(\phi_{s o c}^{\max }\right)$, and a factor that represents the EV SOC at arrival $\left(\phi_{s o c}\right)$.

$$
\begin{aligned}
& \sum_{e} n_{e, v, u}^{e v}=N_{v, u}^{E V}+\phi(\varepsilon) \sigma_{v, u}^{E V} \quad \forall v, u \\
& \sum_{p} P_{e}^{c h} n_{p, e, u}^{c h o} d w^{c s} \geq \sum_{v} n_{e, v, u}^{e v} E_{v}^{r e q}\left(\phi_{s o c}^{\max }-\phi_{s o c}\right) \forall e, u
\end{aligned}
$$

\section{F. Chance Constraints for the Substation Capacity}

Chance constrained programming is a type of robust programming that incorporates randomness in the model via a probabilistic measure over uncertain constraints [19]. The constraints, which contain stochastic parameters, are guaranteed to be satisfied with a certain probability at the optimum solution point. Thus, the chance constraint (30) considers the stochastic behavior of the conventional loads and EV demand, and it guarantees the fulfillment of the substation capacity within a determined confidence level. This constraint is written in terms of the stochastic apparent power supplied by the substation $\left(\widetilde{S}_{s, u}\right)$, the capacity $\left(\bar{S}_{t}\right)$, and the investment variables. It guarantees that the substation capacity is satisfied considering a robustness probability (related to the robustness parameter $\varepsilon$ ).

$$
\operatorname{Prob}\left\{\widetilde{S}_{s, u} \leq \sum_{t} \bar{S}_{t} y_{s, t, u}^{s u b}\right\} \geq 1-\varepsilon \forall s, u
$$

Chance constraint (30) can be represented by the linear constraint (31), as proposed in [19], in which $S_{s, u}$ and $\sigma_{s, u}$ are, respectively, the mean value and the standard deviation of the apparent power supplied by the substation.

$$
S_{s, u}+\phi(\varepsilon) \sigma_{s, u} \leq \sum_{t} \bar{S}_{t} y_{s, t, u}^{s u b} \forall s, u
$$

It is necessary to obtain an expression relating the uncertain load with the apparent power supplied by the substations. Since there is not an explicit relationship between the active power demand and the apparent power of the substation, and due to the nonlinear relationship between active, reactive, and apparent power (given by $S_{i, u}=\sqrt{P_{s, u}^{S}{ }^{2}+Q_{s, u}^{S}}{ }^{2}$ ), the calculation of the mean and the standard deviation of $\widetilde{S}_{s, u}$ is complex. Therefore, these values are calculated by estimating the active power and assuming a power factor for the power supplied by the substation $\left(\phi_{p f}\right)$. For this purpose, the active power supplied by the substation can be expressed in terms of the uncertain demands $\left(\widetilde{P}_{i, u}^{D}\right)$, the EV demand represented by the number of chargers $\left(n_{i, e, u}^{c h o}\right)$ with its corresponding rated power $\left(P_{e}^{c h}\right)$, the power injected by the DG units, and the power losses, as shown in (32). Moreover, it is assumed that the power losses correspond to a percentage of the total active power supplied by the substation $\left(\% P^{\text {loss }}\right)$.

$$
\begin{gathered}
\widetilde{S}_{s, u}=\sum_{i} \omega_{s, i, u}^{s u b}\left[\left(1+\% P^{\text {loss }}\right)\left(\widetilde{P}_{i, u}^{D}+\sum_{e} n_{i, e, u}^{c h o} P_{e}^{c h}\right)\right. \\
\left.-\sum_{g} P_{i, g, u}^{D G}\right] \phi_{p f}^{-1} \forall s, u
\end{gathered}
$$

The binary variable $\omega_{s, i, u}^{s u b}$ indicates whether the node $i$ is connected to the substation $s$; it is obtained from the analytical formulation that finds the shortest path through a radial graph between each node and its corresponding source, as explained in [26].

Since it is assumed that the loads are independent normal variables, the mean value for the apparent power is obtained from (32) by taking the mean values of the demands, as shown in (33).

$$
\begin{aligned}
S_{s, u}= & \sum_{i} \omega_{s, i, u}^{s u b}\left[\left(1+\% P^{\text {loss }}\right)\left(P_{i, u}^{D}+\sum_{e} n_{i, e, u}^{c h o} P_{e}^{c h}\right)\right. \\
& \left.-\sum_{g} P_{i, g, u}^{D G}\right] \phi_{p f}^{-1} \forall s, u
\end{aligned}
$$

On the other hand, the standard deviation for the apparent power is calculated by (34)-(35), considering that, for normal distribution functions, the variance of the apparent power $\left(\sigma_{s, u}^{s q}\right)$ corresponds to the sum of the load variances $\left(\sigma_{i, u}^{D}{ }^{2}\right)$ and that the variance $\left(\sigma_{s, u}^{s q}\right)$ is the square of the standard deviation $\left(\sigma_{s, u}\right)$, (approximated using the function $f$ and a maximum value $\bar{\sigma}_{s, u}$ ).

$$
\begin{aligned}
\sigma_{s, u}^{s q} & =\left[(1+\% \text { Ploss }) \phi_{p f}^{-1}\right]^{2} \sum_{i}{\sigma_{i, u}^{D}}^{2} \omega_{s, i, u}^{s u b} \forall s, u \\
\sigma_{s, u}^{s q} & =f\left(\sigma_{s, u}, \bar{\sigma}_{s, u}, \Gamma\right) \forall s, u
\end{aligned}
$$

Thus, the proposed MILP model, described by (1)-(29), (31), and (33)-(35), is a robust formulation for the multistage joint expansion planning of EDSs and EVCSs, which considers the stochastic behavior of the conventional loads and EV demand. This MILP model can be solved using classical optimization techniques to find the optimal solution that guarantees the fulfillment of the substation capacity within a robustness level.

\section{Test AND RESUlts}

The mathematical model described in Section III was implemented in AMPL [21] and solved via CPLEX [22]. The application of the proposed model is illustrated using a didactic 18-node distribution system adapted from [9] and 54-node distribution system [27].

\section{A. Expansion Planning for the 18-Node Distribution System}

The didactic 18-node distribution system has 4 substations, 14 load nodes, and 26 circuits, and a nominal voltage of $20 \mathrm{kV}$. Two substations of type 1 are constructed in nodes 15 and 16 at the beginning of the planning horizon, as shown in Fig. 1. Furthermore, continuous lines represent constructed circuits, while dashed lines represent circuits for expansion and red numbers correspond to the circuit length. Three planning stages are 


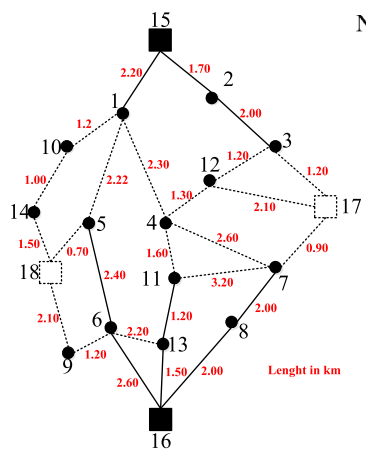

\begin{tabular}{|c|c|c|c|}
\hline \multicolumn{4}{|c|}{ NOMINAL LOAD DATA (KVA } \\
\hline \multirow{2}{*}{ Node } & \\
\hline & 1 & 2 & 3 \\
\hline 1 & 4050 & 4735 & 5420 \\
\hline 2 & 780 & 995 & 1210 \\
\hline 3 & 2580 & 3380 & 3980 \\
\hline 4 & 320 & 410 & 490 \\
\hline 5 & 280 & 370 & 470 \\
\hline 6 & 1170 & 1305 & 1440 \\
\hline 7 & 4040 & 4200 & 4360 \\
\hline 8 & 720 & 830 & 940 \\
\hline 9 & 0 & 1150 & 1350 \\
\hline 10 & 0 & 3050 & 3160 \\
\hline 11 & 1620 & 1620 & 2200 \\
\hline 12 & 0 & 0 & 1220 \\
\hline 13 & 2160 & 2160 & 2400 \\
\hline 14 & 0 & 0 & 2100 \\
\hline
\end{tabular}

Fig. 1. Initial Topology of the 18-node System.

TABLE I

SUBSTATION AND CIRCUIT COSTS $\left(10^{3} \$\right)$

\begin{tabular}{lcccr}
\hline \hline & \multicolumn{2}{c}{$\begin{array}{c}\text { Substations } \\
\text { Final type }\end{array}$} & \multicolumn{2}{c}{$\begin{array}{c}\text { Circuits } \\
\text { Final type }\end{array}$} \\
\hline Initial type & 1 & 2 & 1 & 2 \\
\hline 0 (not build) & 1000 & 1800 & 25 & 35 \\
\hline 1 & - & 800 & - & 30 \\
\hline \hline
\end{tabular}

considered, each one with a duration of 5 years; the load data for each stage is shown in Fig. 1.

Two substation types are available for this system, with capacities of $8 \mathrm{MVA}$ and $12 \mathrm{MVA}$, while two circuit types are considered, with capacities of 197 A and 314 A [9], [20]. The construction and reinforcement costs of the substations and circuits are shown in Table I. The interest rate is defined as $10 \% . c_{s}^{v}$ is 0 , i.e., the operational costs of the substations are neglected. One type of DG unit is considered, with a cost equal to $\$ 2200 \cdot 10^{3}$, a capacity of $3000 \mathrm{kVA}$, and a power factor of 0.95 . The candidate nodes for allocating DG units are $\{1,3,7,8,9,10,11,12\}$, and a limit of $35 \%$ for the DG penetration is adopted. The capacitor allocation considers a limit of six CBs, with at most four modules per bank; parameters $c^{c b}, c^{m o d}$, and $Q_{b c}^{e s p}$ are $\$ 1000$, $\$ 900$, and $300 \mathrm{kVAr}$, respectively.

Two types of EVs are considered to represent the whole EV population: a Tesla and a Nissan Leaf, with battery capacities of $50 \mathrm{kWh}$ and $25 \mathrm{kWh}$. Moreover, the factor $\phi_{\text {soc }}$ is assumed to be 0.5 , based on the probability distribution function for the initial EV SOC presented in [28]. The EV penetration level, i.e., the percentage of users with an $\mathrm{EV}$, is $2 \%, 11 \%$, and $30 \%$ for each stage of the planning horizon [15]. It is also assumed that $20 \%$ of the EVs are charged at home, while the other $80 \%$ are charged in public EVCSs. Therefore, 210, 1688, and $5521 \mathrm{EVs}$ along the three stages are charged in the EVCSs.

The installation cost of an EVCS, including the operational cost, is defined as $c^{c s}=\$ 500$ (the operational cost is considered to be $10 \%$ of the installation cost [29]). The operation time of the EVCSs is defined as 12 hours per day. The candidate nodes for allocating EVCSs are $\{3,8,9,10,11\}$. These locations should have suitable characteristics related to the space and support from nearby residents, as mentioned in [5]. Two types
TABLE II

SUMMARY INVESTMENT AND OPERATIONAL COSTS $\left(10^{3} \$\right)$

\begin{tabular}{lccc}
\hline \hline COST & Case A & Case B & Case C \\
\hline IS & $1,006.46$ & 385.54 & $1,006.46$ \\
IC & 397.74 & 355.32 & 339.11 \\
ICB & 21.79 & 23.39 & 24.22 \\
IDG & $7,462.42$ & $5,766.03$ & $5,766.03$ \\
ICS & 0.00 & $2,324.73$ & $2,875.22$ \\
EC & $67,111.42$ & $58,318.91$ & $59,326.24$ \\
Total & $75,999.83$ & $67,173.91$ & $69,337.29$ \\
\hline \hline
\end{tabular}

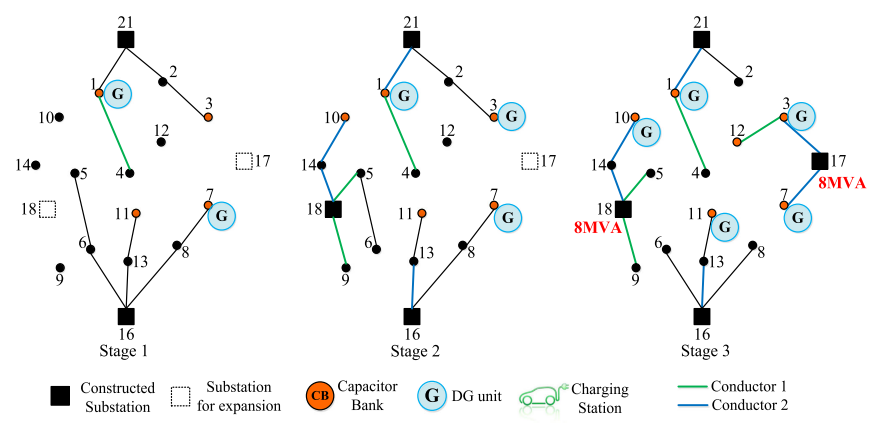

Fig. 2. Multistage EDS expansion planning for Case A.

of chargers, denoted as FC (fast charger), with $c_{e}^{c}=60$ US $\$$ and $P_{e}^{c h}=50 \mathrm{~kW}$, and SC (slow charger), with $c_{e}^{c}=9$ US $\$$ and $P_{e}^{c h}=10 \mathrm{~kW}$, are used in the EVCSs [29], [30].

The results for the EDS expansion planning are analyzed considering a deterministic and robust approach. First, the proposed model is evaluated using deterministic values for the demands, i.e., the corresponding standard deviations are zero. Then, future uncertainties related to the demands are taken into account by the robust formulation.

1) Deterministic Approach: In order to analyze the multistage joint expansion planning of EDSs and EVCSs from a deterministic approach, two cases are evaluated: expansion planning without EVCSs (Case A) and expansion planning considering the allocation and sizing of EVCSs (Case B). In Case A, it is assumed that all of the EVs are charged in homes (i.e., home charging mode) at the peak hour, assuming the worst case for the EDS operation. Table II shows a summary of the investment and operational costs for each case. It can be noted that there is a positive impact on the EDS, due to the allocation of EVCSs. When the EV demand is distributed in each node of the EDS (i.e., Case A), the conventional load is increased, and additional investments are required to meet the total demand. Indeed, Case A presents higher investment costs in substations, circuits, and DG units, as well as in the the energy cost supplied by the substations and the DG units along the planning horizon. In Case B, wherein the EV demand is concentrated in some nodes of the system (i.e., EV charging in EVCSs), the investment and operational costs are reduced by $3.12 \%$ approximately, resulting in a positive impact for the EDS in comparison with Case A. The expansion plans for both cases are illustrated in Figs. 2 and 3 (topology in operation is shown). Note that, without EVCSs (Fig. 2), the 

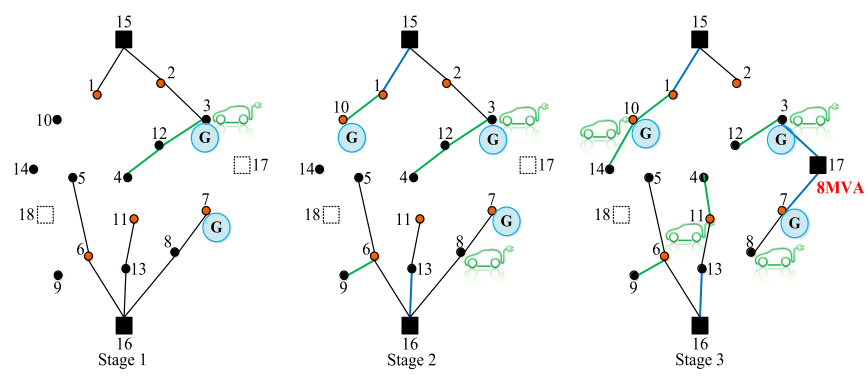

TABLE V

Failure Rate of the Substation CAPACity $(\%)$

\begin{tabular}{|c|c|c|c|c|c|c|c|c|c|c|c|c|}
\hline \multirow[b]{2}{*}{ Substation } & \multicolumn{4}{|c|}{ Stage 1} & \multicolumn{4}{|c|}{ Stage 2} & \multicolumn{4}{|c|}{ Stage 3} \\
\hline & 15 & 16 & 17 & 18 & 15 & 16 & 17 & 18 & 15 & 16 & 17 & 18 \\
\hline Case B & 0.0 & 0.2 & - & - & 3.9 & 67.9 & - & - & 43.8 & 36.6 & 0.4 & - \\
\hline Case C & 0.0 & 1.4 & - & - & 0.0 & 0.0 & 0.2 & - & 0.0 & 2.6 & 2.2 & 1.0 \\
\hline
\end{tabular}

Fig. 3. Multistage EDS expansion planning for Case B.

TABLE III

NUMBER OF CHARGERS: DETERMINISTIC APPROACH

\begin{tabular}{|c|c|c|c|c|c|c|}
\hline \multirow{2}{*}{$\begin{array}{l}\text { Stage } \\
\text { Node }\end{array}$} & \multicolumn{2}{|c|}{1} & \multicolumn{2}{|c|}{2} & \multicolumn{2}{|c|}{3} \\
\hline & $\mathrm{FC}$ & $\mathrm{SC}$ & FC & SC & FC & $\mathrm{SC}$ \\
\hline 3 & 2 & 9 & 14 & 7 & 23 & 187 \\
\hline 8 & - & - & 7 & 1 & - & - \\
\hline 14 & - & - & - & - & 1 & 3 \\
\hline 15 & - & - & - & - & - & 23 \\
\hline Total & 2 & 9 & 21 & 8 & 24 & 213 \\
\hline
\end{tabular}

TABLE IV

NUMBER OF CHARGERS: ROBUST APPROACH

\begin{tabular}{|c|c|c|c|c|c|c|}
\hline \multirow{2}{*}{$\begin{array}{l}\text { Stage } \\
\text { Node }\end{array}$} & \multicolumn{2}{|c|}{1} & \multicolumn{2}{|c|}{2} & \multicolumn{2}{|c|}{3} \\
\hline & $\mathrm{FC}$ & SC & $\mathrm{FC}$ & $\mathrm{SC}$ & $\mathrm{FC}$ & $\mathrm{SC}$ \\
\hline 3 & - & - & 6 & - & - & 52 \\
\hline 8 & 3 & 8 & 2 & - & 16 & 41 \\
\hline 13 & - & - & - & - & 3 & 214 \\
\hline 14 & - & - & 20 & 1 & 2 & 6 \\
\hline Total & 3 & 8 & 28 & 1 & 21 & 313 \\
\hline
\end{tabular}

substations at nodes 17 and 18 are constructed in Stage 3, and most of the circuits are built with higher capacity conductors.

In Case B, when EVCSs are considered, the topology of the network is different. Substation 17 is constructed in Stage 3, and different circuits are constructed/reinforced using, mostly, conductors with lower capacity (see Fig. 3). Furthermore, the allocation of the DG units and CBs is also different when the EVCSs are considered in the expansion planning. Only three DG units are installed in Stage 3 for Case B, while for Case A, five DG units are required in the same stage. The EVCSs are mainly allocated at nodes near the DG units and CBs, in an attempt to maintain a suitable operation of the EDS. For instance, one EVCS is located at node 3, along with a DG unit in Stage 1, and another EVCS and a CB are located at node 11 in Stage 3.

Table III shows the number of each type of charger allocated in each node and each stage for Case B. Four EVCSs and 277 chargers ( $47 \mathrm{FCs}$ and $230 \mathrm{SCs}$ ) are necessary to meet the power demand of the EVs during the planning horizon.

Finally, it must be highlighted that, in both cases, some circuits are disconnected in order to maintain the radial operation of the EDS.

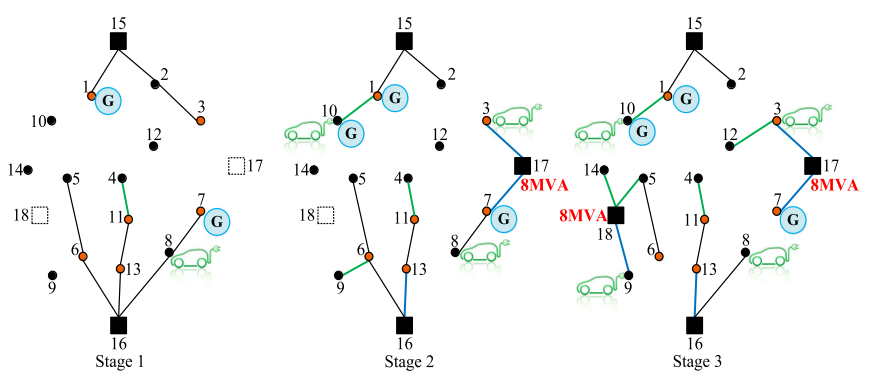

Fig. 4. Multistage EDS expansion planning for Case C.

2) Robust Approach: The multistage joint expansion planning of the EDS and EVCSs is analyzed considering the stochastic behavior of the conventional loads and EV demand. Case B is extended, and the expansion planning, considering allocation and sizing of the EVCSs, is solved using the proposed robust formulation (Case C). It is assumed that the conventional load and the number of EVs follow a normal distribution. The mean values for the loads are the ones shown in Fig. 1, while the mean values for the number of EVs are the same as those in Case B. Moreover, the standard deviations are equal to $15 \%$ of the corresponding mean values.

The robustness parameter used in the chance constraint for the substation capacity and the estimation of the number of EVs is $5 \%$, i.e., $\phi(\varepsilon)$ is equal to 1.645 . This value guarantees the accomplishment of the substation capacity constraint with a probability of $95 \%$. Furthermore, it covers $95 \%$ of the area under the normal distribution curve for the EV penetration.

The investment and operational costs for Case $\mathrm{C}$ are shown in Table II. It should be noted that the total cost of the robust solution is higher than the total cost of the deterministic solution (Case B), a difference of approximately $4 \%$. This fact is due to the additional investments required to guarantee enough capacity of the substations, in order to account for uncertainties in the demand.

In Case $\mathrm{C}$, two new substations are constructed along the planning horizon: Substations 17 and 18 in Stages 2 and 3, respectively (see Fig. 4). It must be highlighted that, in comparison with Case B, one additional substation should be built to avoid possible overloads related to demand growth that may be larger than expected. It should also be noted that the investments in the circuits and the topology of the EDS are different in comparison with Case B, i.e., circuits of higher capacities are used. For instance, conductors of type 2 are necessary to connect some EVCSs. 


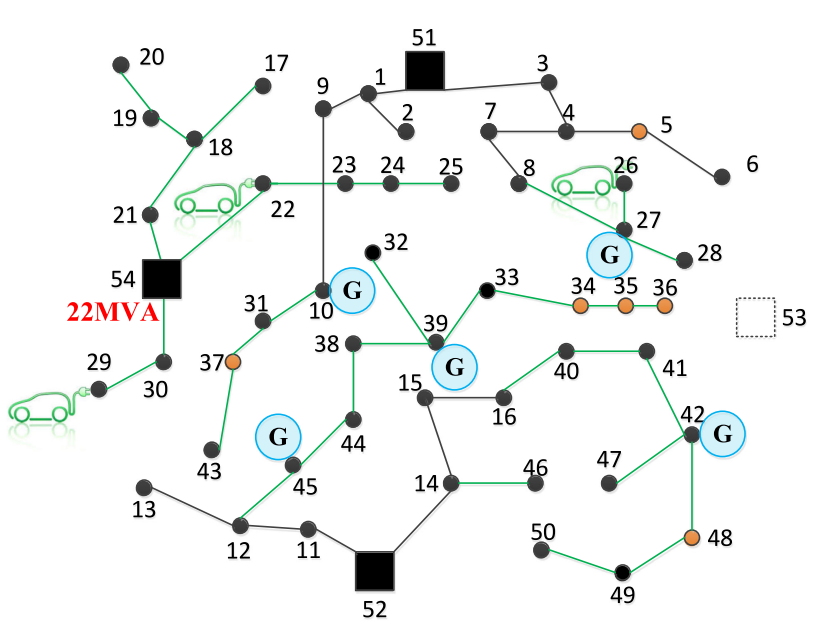

(a)

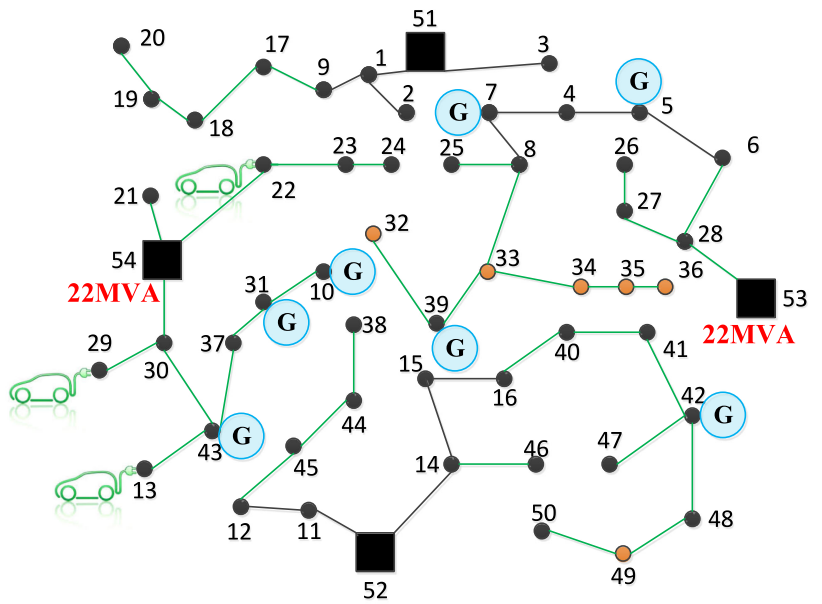

(b)

Fig. 5. Multistage EDS expansion planning for 54-node system at stage 3. (a) Case B and (b) Case C.

The allocation of DG units and CBs is also different along the expansion horizon when uncertainty is considered. The CBs are allocated at the same nodes as in Case A. However, three DG units are allocated at nodes 1,7, and 10. The allocation and sizing of the EVCSs for Case C are shown in Table IV. Four EVCSs with 374 chargers ( $52 \mathrm{FCs}$ and $322 \mathrm{SCs}$ ) are necessary to meet the power demand of the EVs during the planning horizon. As in Case B, most of the EVCSs are allocated at nodes close to energy sources and CBs.

The results show that the expansion plans for the deterministic and robust approaches (Case B and C, respectively) are different in terms of the investment costs in substations, circuits, CBs, and EVCSs. Those costs are increased in order to provide a robust solution for the EDS expansion planning. In all cases, the results show changes in the topology of the network, in the reinforcement of circuits, and in the construction of new substations. These investments are proposed to satisfy the conventional loads and EV demand requirements.

Additionally, Monte Carlo simulations are carried out in order to evaluate the robustness of the expansion plan, considering the substation capacity. The simulations are made taking into account the normal distribution of the stochastic demands. Table V shows the failure rate of the substation capacity for Cases B and $\mathrm{C}$ at each stage of the planning horizon. The results obtained after 1,000 Monte Carlo simulations show that the deterministic approach (Case B) has the worst performance, with violations in the substation capacities, greater than $10 \%$. Specifically, at node 16, the violation is higher than $65 \%$ in Stage 2 and almost $37 \%$ in Stage 3. Although the solution for Case C has a larger cost, the failure rate of the substation capacity is maintained below the limit defined by the robustness parameter, i.e., lower than $5 \%$.

The results show that, considering only economic aspects, the best expansion plan is the one obtained using the deterministic approach, i.e., Case B. However, under uncertain conventional load and EV demand, this plan presents a large substation capacity failure rate. On the other hand, the more expensive investment plan, found by the robust approach (Case C), is robust enough to deal with the uncertainty associated with the demands.

\section{B. Expansion Planning for the 54-Node Distribution System}

An additional test using a 54-node distribution system, adapted from [27] was used in order to verify the scalability of the proposed mathematical model. It was verified that the proposed model is able to solve the complex expansion planning for this distribution system. The results for the EDS expansion planning are analyzed considering the allocation and sizing of the EVCSs, i.e., Case B and Case C (the deterministic and robust approach, respectively).

The investment and operational costs for Case B and Case C are $\$ 83,156.71 \times 10^{3}$ and $\$ 88,668.59 \times 10^{3}$, respectively. As in the 18-node test system, it should be noted that the total cost of the robust solution is higher than the total cost of the deterministic solution (a difference of approximately 6\%). This fact is due to the additional investments required to guarantee enough capacity of the substations to deal with the uncertainties in the demand. In Case B, Substation 54 is built in Stage 1, and different circuits are constructed using only one conductor type. Furthermore, only five DG units are installed in Stage 3 for Case B, while for Case C, seven DG units are required in the same stage (see Fig. 5). The EVCSs are mainly allocated at nodes near the DG units and CBs, in an attempt to maintain a suitable operation of the EDS. In Case C, two new substations are constructed along the planning horizon: Substations 54 and 53 in Stages 2 and 3, respectively. In comparison with Case $\mathrm{B}$, one additional substation should be built to avoid possible technical problems caused by the unexpected demand growth.

The investments in the circuits and the topology of the EDS are also different for both cases. It must be highlighted that, in comparison with Case B, the investment in the circuits in Case $\mathrm{C}$ is also higher. The allocation of DG units and CBs, as well as the EVCSs, is also different along the expansion horizon when uncertainty is considered. For instance, three EVCSs with 792 SCs are necessary to meet the power demand of the EVs during the planning horizon in Case $\mathrm{B}$, whereas in Case C, three EVCSs with 979 chargers (2 FCs and 977 SCs) are installed. As in the previous test, most of the EVCSs are allocated at nodes close to energy sources and CBs. In addition, the robustness of the expansion plan is evaluated for Case $\mathrm{C}$ through Monte 
Carlo simulations. Similar to the previous test system results, the failure rate of the substation capacity is maintained below the limit defined by the robustness parameter.

\section{CONCLUSION}

A novel mixed-integer linear programming (MILP) model for the robust multistage joint expansion planning problem of electrical distribution systems (EDS) and the allocation and sizing of Electric Vehicles Charging Stations (EVCSs) has been developed. The proposed formulation defines the construction/reinforcement of substations, EVCSs, and circuits, and the allocation of distributed generation units and capacitor banks. Chance constraints were used in the robust formulation to consider the uncertainties associated with the conventional loads and EV demand, guaranteeing the fulfillment of the substation capacity and the $\mathrm{EV}$ requirements within a specified confidence level.

The results demonstrate that the allocation of EVCSs results in a positive impact on the expansion plan for the EDS. The impact is mainly reflected by the differences between the substation and the circuit investments, as well as in the topology of the network. Thus, there is a reduction in the total cost of the investment plan when EVCSs are considered in the EDS expansion planning.

The solution provided by a deterministic approach, considering EVCSs, leads to more economic expansion plans. However, under uncertain conventional load and EV demand, it could present high failure rates for the substation capacity. On the other hand, the robust solution provides an investment plan with a larger cost, but it is robust enough to deal with the uncertainty associated with the demands.

In this way, the decision-maker can choose an expansion plan according to a given risk level associated with the demand variations in the EDS operation.

Monte Carlo simulations were carried out in order to verify the compliance of the proposed chance constraint in the robust formulation. It was found that the proposed robust formulation provides a solution in which the substation capacity and the EV requirements are satisfied within a specified confidence level.

\section{APPENDIX}

A piecewise approximation is used to define a function $f$, which calculates the square value of a variable $\rho$, limited by the interval $[0, \bar{\rho}]$. If the interval is partitioned into $\Gamma$ equally sized blocks, the partition set $\mathcal{P}=\{0, \bar{\rho} / \Gamma, 2 \bar{\rho} / \Gamma, \ldots, \bar{\rho}\}$ would be defined so that every block would have an equal length of $\bar{\rho} / \Gamma$. Considering that $\Delta_{\rho, \gamma}$ is a continuous variable that defines the value of the $n$-th block in partition $\mathcal{P}$, the piecewise linear approximation of $\rho^{2}$ is given by (36)-(40). This type of function has a general structure, as follows:

$$
\begin{aligned}
& f(\rho, \bar{\rho}, \Gamma)=\sum_{\gamma=1}^{\Gamma} m_{\rho, \gamma} \Delta_{\rho, \gamma} \\
& \rho^{+}-\rho^{-}=\rho \\
& \rho^{+}+\rho^{-}=\sum_{\gamma=1}^{\Gamma} \Delta_{\rho, \gamma}
\end{aligned}
$$

$$
\begin{aligned}
& 0 \leq \Delta_{\rho, \gamma} \leq \bar{\rho} / \Gamma \quad \forall \gamma=1, . ., \Gamma \\
& m_{\rho, \gamma}=(2 \gamma-1) / \Gamma \quad \forall \gamma=1, . ., \Gamma
\end{aligned}
$$

where $\Gamma$ is the number of discretizations used in the function $f$; $m_{\rho, \gamma}$ is the slope of the $\gamma t h$ block of the piecewise discretization of $\rho ; \Delta_{\rho, \gamma}$ is the value of the $\gamma t h$ auxiliary variable used in the discretization of $\rho$; and $\rho^{+}$and $\rho^{-}$are positive auxiliary variables used in the calculation of $|\rho|$.

\section{REFERENCES}

[1] H. Bulkeley, M. Paterson, and J. Stripple, Towards a Cultural Politics of Climate Change: Devices, Desires and Dissent. Cambridge U.K.: Cambridge Univ. Press, 2016.

[2] M. C. Falvo, D. Sbordone, I. S. Bayram, and M. Devetsikiotis, "EV charging stations and modes: International standards," in Proc. Int Symp. Power Electron., Elect. Drives, Autom. Motion, Jun. 2014, pp. 1134-1139.

[3] K. Clement-Nyns, E. Haesen, and J. Driesen, "The impact of charging plug-in hybrid electric vehicles on a residential distribution grid," IEEE Trans. Power Syst., vol. 25, no. 1, pp. 371-380, Feb. 2010.

[4] P. S. Georgilakis and N. D. Hatziargyriou, "A review of power distribution planning in the modern power systems era: Models, methods and future research," Elect. Power. Syst. Res., vol. 121, pp. 89-100, 2015.

[5] Y. Zheng, Z. Y. Dong, Y. Xu, K. Meng, J. H. Zhao, and J. Qiu, "Electric vehicle battery charging/swap stations in distribution systems: Comparison study and optimal planning," IEEE Trans. Power Syst., vol. 29, no. 1, pp. 221-229, Jan. 2014.

[6] Z. Hu and Y. Song, "Distribution network expansion planning with optimal siting and sizing of electric vehicle charging stations," in Proc. 47th Int Conf. Univ. Power Eng., Sep. 2012, pp. 1-6.

[7] W. Yao et al., "A multi-objective collaborative planning strategy for integrated power distribution and electric vehicle charging systems," IEEE Trans. Power Syst., vol. 29, no. 4, pp. 1811-1821, Jul. 2014.

[8] S. Wang et al., "A stochastic collaborative planning approach for electric vehicle charging stations and power distribution system," in Proc. 2016 IEEE Power Energy Soc. Gen. Meet., Jul. 2016, pp. 1-5.

[9] A. Tabares, J. Franco, M. Lavorato, and M. Rider, "Multistage long-term expansion planning of electrical distribution systems considering multiple alternatives," IEEE Trans. Power Syst., vol. 31, no. 3, pp. 1900-1914, May 2016

[10] N. Neyestani, M. Damavandi, M. Shafie-khah, J. Contreras, and J. Catalao, "Allocation of plug-in vehicles' parking lots in distribution systems considering network-constrained objectives," IEEE Trans. Power Syst., vol. 30, no. 5, pp. 2643-2656, Sep. 2015.

[11] Z. Liu, F. Wen, and G. Ledwich, "Optimal planning of electric-vehicle charging stations in distribution systems," IEEE Trans. Power Del., vol. 28 , no. 1, pp. 102-110, Jan. 2013.

[12] M. Moradijoz, M. P. Moghaddam, M. Haghifam, and E. Alishahi, "A multi-objective optimization problem for allocating parking lots in a distribution network," Int. J. Elect. Power Energy Syst., vol. 46, pp. 115-122, 2013.

[13] F. Fazelpour, M. Vafaeipour, O. Rahbari, and M. A. Rosen, "Intelligent optimization to integrate a plug-in hybrid electric vehicle smart parking lot with renewable energy resources and enhance grid characteristics," Energy Convers. Manage, vol. 77, pp. 250-261, 2014.

[14] M. Amini and A. Islam, "Allocation of electric vehicles' parking lots in distribution network," in Proc. IEEE PES Conf. Innov. Smart Grid Technol., Feb. 2014, pp. 1-5.

[15] I. Frade, A. Ribeiro, G. Gonçalves, and A. Antunes, "Optimal location of charging stations for electric vehicles in a neighborhood in Lisbon, Portugal," Transp. Res. Rec, J. Transp. Res. Board, vol. 2252, no. 12, pp. 91-98, 2011.

[16] L. Zi-fa, W. Zhang, J. Xing, and L. Ke, "Optimal planning of charging station for electric vehicle based on particle swarm optimization," in Proc. IEEE Innov. Smart Grid Technol. Asia, May 2012, pp. 1-5.

[17] E. Pashajavid and M. A. Golkar, "Optimal placement and sizing of plug in electric vehicles charging stations within distribution networks with high penetration of photovoltaic panels," J. Renew. Sustain. Energy, vol. 5 , no. 5, 2013, Art. no. 053126.

[18] S. Guo and H. Zhao, "Optimal site selection of electric vehicle charging station by using fuzzy TOPSIS based on sustainability perspective," Appl. Energy, vol. 158, pp. 390-402, 2015. 
[19] A. Charnes and W. Cooper, "Deterministic equivalents for optimizing and satisficing under chance constraints," Oper. Res., vol. 11, no. 1, pp. 18-39, Jan. 1963.

[20] J. Franco, M. Rider, and R. Romero, "Robust multi-stage substation expansion planning considering stochastic demand," IEEE Trans. Power Syst., vol. 31, no. 3, pp. 2125-2134, May 2016.

[21] R. Fourer, D. M. Gay, and B. W. Kernighan, AMPL: A modeling language for mathematical programming, 2nd ed. Pacific Grove, CA, USA Brooks/Cole-Thomson Learn., 2003.

[22] CPLEX Optimization Subroutine Library Guide and Reference, CPLEX Division ed., ILOG, Inc., Incline Village, NV, USA, 2008.

[23] Z. Liu, F. Wen, and G. Ledwich, "Optimal siting and sizing of distributed generators in distribution systems considering uncertainties," IEEE Trans. Power Del., vol. 26, no. 4, pp. 2541-2551, Oct. 2011

[24] S. Miloca, N. Volpi, J. Yuan, and C. Pinto, "Expansion planning problem in distribution systems with reliability evaluation: An application in real network using georeferenced database," Int. J. Elect. Power Energy Syst., vol. 70, pp. 9-16, 2015.

[25] H. Xing, H. Cheng, L. Zhang, S. Zhang, and Y. Zhang, "Second-order cone model for active distribution network expansion planning," in Proc. 2015 IEEE Power Energy Soc. Gen. Meet., Jul. 2015, pp. 1-5.

[26] J. C. López, M. Lavorato, J. F. Franco, and M. J. Rider, "Robust optimisation applied to the reconfiguration of distribution systems with reliability constraints," IET Gener., Transmiss., Distrib., vol. 10, no. 4, pp. 917-927, 2016.

[27] M. Lavorato, M. J. Rider, A. V. Garcia, and R. Romero, "A constructive heuristic algorithm for distribution system planning," IEEE Trans. Power Syst., vol. 25, no. 3, pp. 1734-1742, Aug. 2010.

[28] J. Quirós-Tortós, L. F. Ochoa, and B. Lees, "A statistical analysis of EV charging behavior in the UK," in Proc. 2015 IEEE PES Innov. Smart Grid Technol. Latin Amer, Oct. 2015, pp. 445-449.

[29] "How much do public and home EV charging stations really cost?" Inside EVs. 2014. [Online]. Available: http://insideevs.com/how-much-dopublic-and-home-ev-charging-stations-really-cost/

[30] Model charging, Tesla Motors, San Carlos, CA, USA. 2017. [Online] Available: http://www.teslamotors.com/models-charging\#/basics

Nataly Bañol Arias received the B.Sc. degree in production engineering from the Universidad Tecnológica de Pereira, Pereira, Colombia, in 2012, and the M.Sc. degree in electrical engineering, in 2015, from São Paulo State University, Ilha Solteira, Brazil, where she is currently working toward the Ph.D. degree in electrical engineering. Her research interests include development of methodologies for the optimization, planning, and control of electrical power systems.
Alejandra Tabares received the B.Sc. degree in production engineering from the Universidad Tecnológica de Pereira, Pereira, Colombia, in 2012, and the M.Sc. degree in electrical engineering, in 2015, from São Paulo State University, Ilha Solteira, Brazil, where she is currently working toward the Ph.D. degree in electrical engineering. Her research interests include the development of methodologies for the optimization, planning, and control of electrical power systems.

John F. Franco (S'11-M'13) received the B.Sc. and M.Sc. degrees from the Universidad Tecnológica de Pereira, Pereira, Colombia, in 2004 and 2006, respectively, and the Ph.D. degree from São Paulo State University (UNESP), Ilha Solteira, Brazil, in 2012, all in electrical engineering. He is currently a Professor at UNESP, Rosana, Brazil. His research interests include development of methodologies for the optimization, planning, and control of electrical power systems.

Marina Lavorato (S'07-M'11) received the B.Sc. and M.Sc. degrees from the Federal University of Juiz de Fora, Juiz de Fora, Brazil, in 2002 and 2004, respectively, and the Ph.D. degree from the University of Campinas, Campinas, Brazil, in 2010, all in electrical engineering. She is currently a Professor in the Electrical Engineering Faculty, Pontifical Catholic University of Campinas, Campinas, Brazil. Her research interests include the development of methodologies for the optimization, planning, and control of electrical power systems.

Rubén Romero (M'93-SM'08) received the B.Sc. and P.E. degrees from the National University of Engineering, Lima, Perú, in 1978 and 1984, respectively, and the M.Sc. and Ph.D. degrees from the University of Campinas, Campinas, Brazil, in 1990 and 1993, respectively, all in electrical engineering. He is currently a Professor in the Electrical Engineering Department, São Paulo State University, Ilha Solteira, Brazil. His research interests include electrical power systems planning. 